\title{
Resummation of scalar correlator in higher spin black hole background
}

\author{
Matteo Beccaria and Guido Macorini \\ Dipartimento di Matematica e Fisica "Ennio De Giorgi", \\ Università del Salento \& INFN, Via Arnesano, 73100 Lecce, Italy \\ E-mail: matteo.beccaria@le.infn.it, guido.macorini@le.infn.it
}

ABSTRACT: We consider the proposal that predicts holographic duality between certain 2D minimal models at large central charge and Vasiliev 3D higher spin gravity with a single complex field. We compute the scalar correlator in the background of a higher spin black hole at order $\mathcal{O}\left(\alpha^{5}\right)$ in the chemical potential $\alpha$ associated with the spin-3 charge. The calculation is performed at generic values of the symmetry algebra hs $[\lambda]$ parameter $\lambda$ and for the scalar in three different representations. We then study the perturbative data in the Bergshoeff-Blencowe-Stelle limit where $\lambda$ is taken large and discover remarkable regularities. This leads to formulate a conjectured closed formula for the resummation of the various subleading terms at large $\lambda$ up to the order $\mathcal{O}\left(\alpha^{n} \lambda^{2 n-3}\right)$.

KeYwords: Gauge-gravity correspondence, Black Holes in String Theory, AdS-CFT Correspondence

ArXiv ePrint: 1311.5450 


\section{Contents}

1 Introduction 1

2 Higher spin black hole in hs $[\lambda] \oplus$ hs $[\lambda]$ Chern-Simons gravity 3

3 Scalar correlator in higher spin gravity 4

4 Structure of the scalar correlator in the black hole background 5

5 Calculation of $\mathcal{O}\left(\alpha^{5}\right)$ correlators 5

6 Consistency checks $\quad 6$

6.1 Representation constraints 6

$\begin{array}{lll}6.2 & \text { Zero temperature limit } & 7\end{array}$

7 Resummation conjectures in the Bergshoeff-Blencowe-Stelle limit $\quad 8$

$\begin{array}{ll}7.1 \text { Leading order } & 8\end{array}$

$\begin{array}{ll}7.2 \text { Next-to-leading order } & 9\end{array}$

7.3 Next-next-to-leading order 9

$\begin{array}{lll}7.4 & \text { Higher orders } & 10\end{array}$

$\begin{array}{ll}\text { 7.5 Resummation structure in the zero temperature limit } & 10\end{array}$

8 Conclusions 11

A The infinite dimensional algebra hs $[\lambda] \quad 12$

B Non-zero polynomials $p_{m, k}^{(n)}(\lambda)$ for $n \leq 4 \quad 12$

$\begin{array}{lll}\text { B.1 Fundamental representation } & 12\end{array}$

$\begin{array}{ll}\text { B.2 2-Antisymmetric representation } & 15\end{array}$

$\begin{array}{ll}\text { B.3 3-Antisymmetric representation } & 17\end{array}$

C Zero temperature limit for the 2-antisymmetric representation $\quad 19$

D Eigenvalue of $V_{0}^{3}$ on the $K$-antisymmetric representation 20

$\begin{array}{ll}\text { E The resummation functions } f_{N^{3} L O} & 20\end{array}$

\section{Introduction}

In the striking paper [1], Vasiliev higher spin theory on $A d S_{3}$ coupled to a complex scalar [2, $3]$ has been shown to be holographically dual to the conformal 2D coset conformal minimal models $\mathcal{W}_{N}$ at large $N$. The precise limit that is considered has a large central charge 
and is parametrised by a single constant $\lambda$ whose gravity meaning is that of labelling a family of $A d S_{3}$ vacua. The symmetry content of the two sides of the correspondence matches. In particular, the asymptotic symmetry algebra of the higher spin gravity theory is $\mathcal{W}_{\infty}[\lambda][4-7]$ which is also the classical symmetry of the dual CFT $[1,4]$.

The bulk theory admits higher spin generalisations of the BTZ black hole [8,9] as reviewed in [10]. The thermodynamical properties of these very interesting objects are in agreement with the proposed duality [11, 12]. However, these tests do not probe the dynamics of the higher spin gravity complex scalar that plays no role in matching the black-hole entropy. Indeed, black holes in $A d S_{3}$ are universal and explore the CFT thermodynamics at high temperature, which in two dimensions is only determined by the chiral algebra and is insensitive to the microscopic features of the conformal theory.

The quantum fluctuations of the complex scalar have been studied in [13]

by computing the bulk-to-boundary scalar propagator in the background of the $3 \mathrm{D}$ higher spin black hole analysed in [11], at least to first order in the spin-3 charge chemical potential $\alpha$ and for a special value of the $\lambda$ parameter. Later, the authors of [14], extended the calculation in several directions. They pushed the calculation to order $\mathcal{O}\left(\alpha^{2}\right)$, extended it to all $\lambda$, and also considered the two-box antisymmetric representation. Remarkably, they demonstrated explicitly the agreement with a CFT calculation.

In this paper, we considered again the correlator for the scalar field transforming in three different representations of the higher spin algebra. To be precise, this means that one assigns a higher representation $\Lambda_{+}$of the symmetry algebra to the scalar dual CFT primary. In the bulk, the Vasiliev master field $C$ obeys the equation $d C+A C-C \bar{A}=0$ where the higher spin gauge connections $A, \bar{A}$ are in the representation $\Lambda_{+}$. This implies that, in the bulk, the master field $C$ actually corresponds to an infinite tower of fields with different masses. The details of the spectrum can be worked out by decomposing $\Lambda_{+}$ into irreducible representations of $\mathfrak{s l}(2)$ as discussed in [15]. In each case, we computed the propagator at order $\mathcal{O}\left(\alpha^{5}\right)$, by applying the powerful methods introduced in $[13,14]$. The reason for such a brute-force calculation is the idea that some regularity could be observable once we have enough perturbative data to inspect.

In particular, we looked for special features of the large $\lambda$ regime in the spirit of the analysis in [16]. The large $\lambda$ limit of the algebra hs $[\lambda]$ has been considered in the literature in [17] and is the algebra of area-preserving diffeomorphisms of a $2 \mathrm{~d}$ hyperboloid. In the following, we shall denote this limit as the Bergshoeff-Blencowe-Stelle limit after the names of the authors of [17]. The mathematical reason why this limit is potentially interesting is that for $\lambda=-\mathcal{N}$, a negative integer, the pure higher spin sector of Vasiliev theory reduced to that of a $\mathfrak{s l}(\mathcal{N}) \oplus \mathfrak{s l}(\mathcal{N})$ Chern-Simons 3D gravity theory (the full theory, including the scalar, is of course more complicated). Taking $\mathcal{N}$ to be large could lead to some simplifications in the structure of the perturbative corrections. This attitude proved to be successful in the analysis of the black hole partition function [16] leading to novel exact results. From the physical point of view, the Bergshoeff-Blencowe-Stelle limit is not necessarily relevant. However, it can lead to technical simplified structures that can be helpful in at least two ways. First, as a check of a fully fledged calculation. Second, as a possible controlled approximation order by order to the extent that one can control the $1 / \lambda$ corrections. 
Anyway, although optimistic, this simple attitude turns out to be correct, even beyond the leading order. For the three considered representations of the scalar, we show that all corrections of the order $\mathcal{O}\left(\alpha^{n} \lambda^{2 n-p}\right)$ with $p=0,1,2,3$, i.e. at $N^{3} L O$, can be resummed in closed form consistently with the available perturbative data. Also, the extension of the results to a generic $K$-antisymmetric representation $\left(\square^{\otimes K}\right)_{A}$ appears to be straightforward, once one recognises that the representation dependent feature of the resummation formula is encoded in the spin-3 zero mode eigenvalue of the highest weight of $\left(\square^{\otimes K}\right)_{A}$. Clearly, our resummation expressions stand as open conjectures unless a full analytical proof of exponentiation will be available in the Bergshoeff-Blencowe-Stelle limit.

The plan of the paper is the following. In section (2), we review the formulation of higher spin black holes in hs $[\lambda] \oplus \mathrm{hs}[\lambda]$ Chern-Simons 3D gravity. In section (3), we summarise the methods that allow to evaluate the scalar correlator. In section (4), we present the general structure of the correlator in perturbation theory and in section (5) we give some details of the actual $\mathcal{O}\left(\alpha^{5}\right)$ calculation. The results are non trivially checked in section (6). Finally, section (7) is devoted to our proposed resummation formulae.

\section{Higher spin black hole in hs $[\lambda] \oplus$ hs $[\lambda]$ Chern-Simons gravity}

The gauge fields of hs $[\lambda] \oplus$ hs $[\lambda]$ Chern-Simons gravity in $A d S_{3}$ are $A$ and $\bar{A}$, and obey the equation of motion

$$
d A+A \wedge \star A=0
$$

as well as its conjugate. The generators of hs $[\lambda]$ are usually denoted $V_{m}^{s}$ with $|m|<s$. Space-time is described by a radial coordinate $\rho$ and Euclidean torus coordinates $(z, \bar{z})$ with periodic identification

$$
(z, \bar{z}) \sim(z+2 \pi, \bar{z}+2 \pi) \sim(z+2 \pi \tau, \bar{z}+2 \pi \bar{\tau})
$$

As shown in [18], it is possible to choose a gauge where the black hole solution can be written in the form

$$
A(\rho, z, \bar{z})=b^{-1} a b+b^{-1} d b, \quad \bar{A}(\rho, z, \bar{z})=b \bar{a} b^{-1}+b d b^{-1},
$$

where $b=e^{\rho V_{0}^{2}}$ and $a, \bar{a}$ are constant connections without radial components $a_{\rho}=\bar{a}_{\rho}=0$. The black hole solution with higher spin charges found in [11] has the following explicit expression of the holomorphic connection

$$
\begin{aligned}
& a_{z}=V_{1}^{2}-\frac{2 \pi \mathcal{L}}{k} V_{-1}^{2}-\frac{\pi \mathcal{W}}{2 k} V_{-2}^{3}+\sum_{n=4}^{\infty} \mathcal{J}_{n} V_{-n+1}^{n}, \\
& a_{\bar{z}}=-\frac{\alpha}{\bar{\tau}} a_{z} \star a_{z}-\text { trace. }
\end{aligned}
$$

Here, $\star$ is the lone star product [19], $(\mathcal{L}, \mathcal{W})$ are the stress tensor and spin-3 charges, while $\mathcal{J}_{n}$ are higher spin charges. Finally, $k$ is the Chern-Simons level. The explicit expansion of 
the charges in powers of the chemical potential $\alpha$ associated with the spin- 3 charge have been derived in $[11,16]$. At order $\mathcal{O}\left(\alpha^{5}\right)$, we shall need the following expressions,

$$
\begin{aligned}
\mathcal{L} & =-\frac{k}{8\left(\pi \tau^{2}\right)}+\frac{\alpha^{2} k\left(\lambda^{2}-4\right)}{24 \pi \tau^{6}}-\frac{\alpha^{4}\left(k\left(\lambda^{2}-7\right)\left(\lambda^{2}-4\right)\right)}{24\left(\pi \tau^{10}\right)}+\mathcal{O}\left(\alpha^{6}\right) \\
\mathcal{W} & =-\frac{\alpha k}{3\left(\pi \tau^{5}\right)}+\frac{10 \alpha^{3} k\left(\lambda^{2}-7\right)}{27 \pi \tau^{9}}-\frac{\alpha^{5}\left(k\left(5 \lambda^{4}-85 \lambda^{2}+377\right)\right)}{9\left(\pi \tau^{13}\right)}+\mathcal{O}\left(\alpha^{7}\right) \\
\mathcal{J}_{4} & =\frac{7 \alpha^{2}}{36 \tau^{8}}-\frac{7 \alpha^{4}\left(2 \lambda^{2}-21\right)}{36 \tau^{12}}+O\left(\alpha^{6}\right) \\
\mathcal{J}_{5} & =\frac{5 \alpha^{3}}{18 \tau^{11}}-\frac{\alpha^{5}\left(44 \lambda^{2}-635\right)}{54 \tau^{15}}+\mathcal{O}\left(\alpha^{7}\right) \\
\mathcal{J}_{6} & =\frac{143 \alpha^{4}}{324 \tau^{14}}+\mathcal{O}\left(\alpha^{6}\right) \\
\mathcal{J}_{7} & =\frac{182 \alpha^{5}}{243 \tau^{17}}+\mathcal{O}\left(\alpha^{7}\right) .
\end{aligned}
$$

From the explicit form of the charges, one can derive the thermal partition function and the black hole entropy [20,21].

\section{Scalar correlator in higher spin gravity}

Let us briefly summarise how to compute the scalar bulk-boundary propagator in 3D higher spin gravity, see for instance [13, 15, 22-24]. Our presentation closely follows [14] whose notation we adopt. Assuming the gauge choice (2.3), we define

$$
\begin{array}{ll}
\Lambda_{0}=a_{\mu} x^{\mu}, & \Lambda_{\rho}=b^{-1} \star \Lambda_{0} \star b, \\
\bar{\Lambda}_{0}=\bar{a}_{\mu} x^{\mu}, & \bar{\Lambda}_{\rho}=b \star \bar{\Lambda}_{0} \star b^{-1} .
\end{array}
$$

Then, for a bulk scalar with mass $m^{2}=\Delta(\Delta-2)$ transforming in a representation of hs $[\lambda]$, the bulk-boundary propagator reads

$$
\Phi(z, \bar{z}, \rho ; 0)=e^{\Delta \rho} \operatorname{Tr}\left[e^{-\Lambda_{\rho}} \star c \star e^{\bar{\Lambda}_{\rho}}\right],
$$

where $c$ is a highest weight of hs[ $\lambda]$, i.e. an eigenstate of $V_{0}^{2}$ under star product annihilated by positive modes. The boundary two-point correlator between two dual fields at positive infinity can be extracted by taking the $\rho \rightarrow+\infty$ limit giving from AdS/CFT duality

$$
\Phi(z, \bar{z}, \rho ; 0) \sim e^{-\Delta \rho}\langle\bar{\varphi}(z, \bar{z}) \varphi(0,0)\rangle,
$$

where the r.h.s. is computed in the holographic dual CFT with $\mathcal{W}_{\infty}[\lambda]$ symmetry and $\varphi$ is a scalar primary of conformal dimension $\Delta$ living in the same representation of hs $[\lambda]$ as the bulk scalar field [15]. In the following, we shall consider the defining representation of hs $[\lambda]$ and its antisymmetric powers. Then, $c$ is the projector onto the highest weight state $c=|\mathrm{hw}\rangle\langle\mathrm{hw}|$ that can be built explicitly from the infinite-dimensional matrix realisation of hs $[\lambda]$ (see appendix A). Thus,

$$
\Phi(z, \bar{z}, \rho ; 0)=e^{\Delta \rho}\left\langle\mathrm{hw}\left|e^{\bar{\Lambda}_{\rho}} e^{-\Lambda_{\rho}}\right| \mathrm{hw}\right\rangle
$$


and in the large $\rho$ limit we find $(\mid-$ hw $\rangle$ is the lowest weight state)

$$
\langle\bar{\varphi}(z, \bar{z}) \varphi(0,0)\rangle=\left\langle-\mathrm{hw}\left|e^{-\Lambda_{0}}\right| \mathrm{hw}\right\rangle\left\langle\mathrm{hw}\left|e^{\bar{\Lambda}_{0}}\right|-\mathrm{hw}\right\rangle .
$$

Due to the above factorisation, we shall consider the purely left-moving part without losing any information. In particular, this means that $a_{\bar{z}}$ will play no role in the following.

\section{Structure of the scalar correlator in the black hole background}

For a scalar in a generic representation of hs $[\lambda]$, we have $[13,14]^{1}$

$$
\begin{aligned}
\langle\bar{\varphi}(z, \bar{z}) \varphi(0,0)\rangle & =\left(4 \tau \bar{\tau} \sin \frac{z}{2 \tau} \sin \frac{\bar{z}}{2 \bar{\tau}}\right)^{-\Delta} R(z, \bar{z}), \\
R(z, \bar{z}) & =1+\sum_{n=1}^{\infty} \frac{\alpha^{n}}{\tau^{2 n}} R^{(n)}(z, \bar{z}) .
\end{aligned}
$$

The corrections $R^{(1)}$ and $R^{(2)}$ have been computed in [14] for the fundamental and $\boxminus$ representations. The calculation is done in the bulk and it is matched on the CFT side where the relevant dual quantity is the torus two-point function of a scalar primary in the presence of a deformation of the conformal theory by a holomorphic spin-3 operator.

Here, we perform an order $\mathcal{O}\left(\alpha^{5}\right)$ calculation including also the $\theta$ representation. In all cases, the general structure of $R^{(n)}$ turns out to be the following:

$$
R^{(n)}(z, \bar{z})=\frac{1}{\sin ^{2 n}\left(\frac{\mathcal{Z}}{2}\right)} \sum_{m=0}^{n}(\mathcal{Z}-\overline{\mathcal{Z}})^{m} \sum_{k=0}^{n} p_{m, k}^{(n)}(\lambda) \begin{cases}\sin (k \mathcal{Z}), n+m \text { odd } \\ \cos (k \mathcal{Z}), n+m \text { even }\end{cases}
$$

where $p_{m, k}^{(n)}(\lambda)$ are degree $2 n$ polynomials and the variables $\mathcal{Z}, \overline{\mathcal{Z}}$ are

$$
\mathcal{Z}=\frac{z}{\tau}, \quad \overline{\mathcal{Z}}=\frac{\bar{z}}{\bar{\tau}}
$$

\section{Calculation of $\mathcal{O}\left(\alpha^{5}\right)$ correlators}

The extension of the results obtained in [14] is fully straightforward. Here, we just point out a few details that can be useful in order to increase the efficiency of the calculation. First, one assumes that the functions $p_{m, k}^{(n)}(\lambda)$ are polynomials. Then, the corrections $R^{(n)}$ are evaluated at $\lambda=-\mathcal{N}$ for $\mathcal{N}=3,4, \ldots$ up to a point where $p_{m, k}^{(n)}(\lambda)$ can be consistently fixed. We always pushed the calculation some order further in order to confirm that $p_{m, k}^{(n)}(\lambda)$ are actually polynomials.

At $\lambda=-\mathcal{N}$, we use the finite-dimensional matrix representation of the generators $V_{m}^{s}$. The matrix element $\left\langle-\mathrm{hw}\left|e^{-\Lambda_{0}}\right| \mathrm{hw}\right\rangle$ is evaluated for the scalar transforming in the $K$-antisymmetric representation $\left(\square^{\otimes K}\right)_{A}$ using

$$
\left|\mathrm{hw}_{K}\right\rangle=\frac{1}{\sqrt{K !}}|1\rangle \otimes|2\rangle \otimes \cdots \otimes|K\rangle+\text { signed permutations, }
$$

\footnotetext{
${ }^{1}$ The sum over images required to impose periodicity $(z, \bar{z}) \rightarrow(z+2 \pi, \bar{z}+2 \pi)$ is left implicit.
} 
and computing

$$
\left\langle-\mathrm{hw}_{K}\left|e^{-\Lambda_{0}}\right| \mathrm{hw}_{K}\right\rangle=\sum\left\langle\mathcal{N}\left|e^{-\Lambda_{0}}\right| 1\right\rangle\left\langle\mathcal{N}-1\left|e^{-\Lambda_{0}}\right| 2\right\rangle \cdots\left\langle\mathcal{N}-k+1\left|e^{-\Lambda_{0}}\right| k\right\rangle,
$$

where the sum is over the signed permutations of the labels $\{1, \ldots, k\}$ in the kets.

The most time consuming part of the calculation is the construction of the exponential $e^{-\Lambda_{0}}$. We expand $-\Lambda_{0}=\sum_{n=0}^{\infty} \alpha^{n} X_{n}$ and write the expansion of the exponential as

$$
E(t)=e^{t \sum_{n=0}^{\infty} \alpha^{n} X_{n}}=\sum_{n=0}^{\infty} \alpha^{n} E_{n}(t) .
$$

From

$$
E^{\prime}(t)=-\Lambda_{0} E(t)
$$

we obtain

$$
\begin{aligned}
& E_{0}(t)=e^{t X_{0}} \\
& E_{n}(t)=\int_{0}^{t} E_{0}(t-s) \sum_{m=1}^{n} X_{m} E_{n-m}(s) d s, \quad n \geq 1 .
\end{aligned}
$$

Finally, the desired exponential is recovered by setting $t=1$. The building block $E_{0}(t)$ is efficiently computed by noting that $X_{0}$ is diagonalised exploiting the relation

$$
e^{-\frac{i}{4 \tau} V_{-1}^{2}} e^{2 i \tau V_{1}^{2}}\left(V_{1}^{2}+\frac{1}{4 \tau^{2}} V_{-1}^{2}\right) e^{-2 i \tau V_{1}^{2}} e^{\frac{i}{4 \tau} V_{-1}^{2}}=\frac{i}{\tau} V_{0}^{2},
$$

This change of basis can be done at the beginning and undone before taking matrix elements.

We have computed the polynomials $p_{m, k}^{(n)}(\lambda)$ for $n \leq 5$ for the three representations $\square, \boxminus$, , . The results for $n \leq 4$ are listed in appendix B. ${ }^{2}$

\section{Consistency checks}

\subsection{Representation constraints}

Let us denote by $\widetilde{R}$ the ratio $R$ with the replacement $\alpha \rightarrow-\alpha$. For simplicity, we can set $\overline{\mathcal{Z}}=0$, since that variable is always paired with $\mathcal{Z}$ according to (4.3). We have

$$
\begin{array}{cc}
\mathcal{N}=-1: & R_{\square}=1, \\
\mathcal{N}=-2: & R_{\square}=R_{\boxminus}=1, \\
\mathcal{N}=-3: & R_{\boxminus}=1, \quad R_{\square}=\widetilde{R}_{\square}, \\
\mathcal{N}=-4: & R_{\square}=\widetilde{R}_{\boxminus}, \\
\mathcal{N}=-5: & R_{\boxminus}=\widetilde{R}_{\boxminus} .
\end{array}
$$

These properties are simply understood in terms of equivalences of representations of $\mathfrak{s l}(\mathcal{N})$ at the above special values of $\mathcal{N}$.

\footnotetext{
${ }^{2}$ The explicit polynomials for $n=5$ are available upon request to the authors.
} 


\subsection{Zero temperature limit}

An interesting regime is the zero-temperature limit where $\mu=\alpha / \bar{\tau}$ is fixed and $\tau, \bar{\tau} \rightarrow$ $\infty$. This is the chiral deformation background discussed in $[13,24]$. The constant flat connection reads in this limit

$$
a=V_{1}^{2} d z-\mu V_{2}^{3} d \bar{z}, \quad \bar{a}=V_{-1}^{2} d \bar{z} .
$$

In [14], it has been proved that

$$
\lim _{\substack{\tau, \bar{\tau} \rightarrow \infty \\ \text { fixed } \mu}} R_{\square}=\sum_{n=0}^{\infty}\left(\frac{\mu \bar{z}}{z^{2}}\right)^{n} \frac{\Gamma(2 n+1+\lambda)}{n ! \Gamma(1+\lambda)} .
$$

In appendix $\mathrm{C}$, we prove with a similar calculation that

$$
\lim _{\substack{\tau, \bar{\tau} \rightarrow \infty \\ \text { fixed } \mu}} R_{\boxminus}=\sum_{n=0}^{\infty}\left(\frac{\mu \bar{z}}{z^{2}}\right)^{n} \sum_{m=0}^{n} \frac{(1+\lambda)_{2 m}^{+}(1+\lambda)_{2(n-m)}^{+}}{m !(n-m) !} \frac{\lambda+n+1+2(n-2 m)^{2}}{\lambda+1},
$$

where $(a)_{n}^{+}=a(a+1) \cdots(a+n-1)$ is the ascending Pochammer symbol. The explicit first terms are

$$
\begin{aligned}
& \lim _{\substack{\tau, \bar{\tau} \rightarrow \infty \\
\text { fixed } \mu}} R_{\boxminus}=1+\frac{2(\lambda+2)(\lambda+4) \mu \bar{z}}{z^{2}}+\frac{2(\lambda+2)(\lambda+3)\left(\lambda^{2}+9 \lambda+23\right) \mu^{2} \bar{z}^{2}}{z^{4}} \\
& +\frac{4(\lambda+2)(\lambda+3)(\lambda+4)(\lambda+6)\left(\lambda^{2}+9 \lambda+29\right) \mu^{3} \bar{z}^{3}}{3 z^{6}} \\
& +\frac{2(\lambda+2)(\lambda+3)(\lambda+4)(\lambda+5)\left(\lambda^{4}+22 \lambda^{3}+197 \lambda^{2}+872 \lambda+1641\right) \mu^{4} \bar{z}^{4}}{3 z^{8}}+\cdots
\end{aligned}
$$

We have checked that our explicit expression for $R_{\boxminus}$ obeys this limit at order $\mathcal{O}\left(\mu^{5}\right)$. We remark that the zeroes appearing in the above expression are not explained in terms of a representation theoretic argument. Indeed, they appear also in the case of the fundamental representation and come from the $\Gamma$ function ratios in (C.3). They express the fact that, when $\lambda=-\mathcal{N}$, a negative integer, the matrix elements in (C.3) require $\mathcal{N}$ to be large enough in order to be non zero. This is because expressions of the form $\left(V_{+}\right)^{q}\left(V_{-}\right)^{q}|\mathrm{hw}\rangle$, where $V_{ \pm}$are ladder operators, are trivially zero when $q$ is so large that $|\mathrm{hw}\rangle$ is annihilated by the lowering operator. In particular, as a general pattern, the expansion in (6.9) stops at order $\left(\mu \bar{z} / z^{2}\right)^{\mathcal{N}-2}$ with the term $\left(\mu \bar{z} / z^{2}\right)^{\mathcal{N}-3}$ always vanishing for even $\mathcal{N}{ }^{3}$ The fact that the linear (cubic) term in (6.9) vanishes at $\lambda=4(\lambda=6)$ is a special case of this rule.

\footnotetext{
${ }^{3}$ Technically, this follows from the fact that for $n=\mathcal{N}-3$ and $-\lambda=\mathcal{N}$ even, exactly one of the three factors

$$
(1+\lambda)_{2 m}^{+}, \quad(1+\lambda)_{2(n-m)}^{+}, \quad \lambda+n+1+2(n-2 m)^{2}
$$

appearing in (6.8), does vanish for each $0 \leq m \leq n$. In particular, the third factor vanishes for $m=\frac{\mathcal{N}}{2}-1$ and $m=\frac{\mathcal{N}}{2}-2$ while the other two factors vanish before and after these two consecutive integers, respectively.
} 


\section{Resummation conjectures in the Bergshoeff-Blencowe-Stelle limit}

As we mentioned in the Introduction, an interesting regime where the scalar correlator can be considered is the Bergshoeff-Blencowe-Stelle limit $\lambda \rightarrow \infty$ where hs $[\lambda]$ becomes the algebra of area-preserving diffeomorphisms of a $2 \mathrm{~d}$ hyperboloid [17]. This regime has been recently explored in [16] in the case of the higher spin black hole partition function. One finds that, with a suitable normalisation of hs $[\lambda]$ generators, the large $\lambda$ limit is smooth and non trivial. Indeed, all higher spin charges are turned on and contribute. Here, we shall also find that the correlators have a sensible large $\lambda$ limit. The truncation at $\lambda=-\mathcal{N}$ suggest a possible treatment of this regime. The considered observables have a polynomial dependence on $\lambda$, at least at each order of perturbation theory in $\alpha$. This means that the large $\lambda$ limit in hs $[\lambda]$ is the same as the large $\mathcal{N}$ limit in the $\mathfrak{s l}(\mathcal{N}) \oplus \mathfrak{s l}(\mathcal{N})$ Chern-Simons gravity. One expects that considering $\mathfrak{s l}(\infty)$ could lead to some special properties.

In the present case of the scalar correlator, we have investigated the large $\lambda$ limit and found a remarkable pattern of regularities order by order in the expansion in powers of the spin-3 chemical potential. As a preliminary remark, let us notice that we can set $\overline{\mathcal{Z}}=0$ since this variable can be inserted back by noting that $\overline{\mathcal{Z}}$ always accompany $\mathcal{Z}$ in the combination $\mathcal{Z}-\overline{\mathcal{Z}}$ outside trigonometric functions, see (4.3). The explicit expression of the ratio $R_{r}$, where $r=\square, \boxminus, \boxminus$, admits the exact expansion

$$
R_{r}=1+\sum_{n=1}^{\infty} \sum_{m=0}^{2 n} \rho_{n, m}(\mathcal{Z}) \frac{1}{\lambda^{m}} \frac{\left(\lambda^{2} \alpha\right)^{n}}{\tau^{2 n}} .
$$

Thus, the index $n$ labels the order in $\alpha$, while the index $m$ labels the various subleading terms at large $\lambda$ apart from the factor $\lambda^{2}$ accompanying each factor $\alpha$. By a proposed resummation of (7.1), we mean a formula that is able to capture many contributions in terms of a few $\mathcal{Z}$-dependent coefficients. Of course, we have a limited set of perturbative data (up to $n=5$ ) and we can only suggest a possible resummation formula as a conjecture.

\subsection{Leading order}

A leading order resummation is a formula that is able to capture all the terms in (7.1) with $m=0$. Let us introduce the following coefficient associated with the $K$-antisymmetric representation

$$
c_{r=(\square \otimes K)_{A}}=\frac{K}{6}(\lambda+K)(\lambda+2 K) .
$$

We find that

$$
R_{r} \stackrel{\lambda \rightarrow \infty}{=} \exp \left(\frac{f_{L O}}{\sin ^{2} \frac{\mathcal{Z}}{2}} \frac{c_{r} \alpha}{\tau^{2}}\right)+\mathcal{O}\left(\alpha^{n} \lambda^{2 n-1}\right)
$$

where

$$
f_{L O}=-\mathcal{Z}+\frac{3 \sin \mathcal{Z}}{2}-\frac{1}{2} \mathcal{Z} \cos \mathcal{Z} .
$$

Notice that a single coefficient function $f_{L O}$ is enough to capture all available terms $\rho_{n, 0}$ in (7.1). The function $f_{L O}$ can be identified with the associated term in the $\mathcal{O}(\alpha)$ contribution to $R_{r}$. From the CFT calculation of the $\mathcal{O}(\alpha)$ contribution to $R$, we identify the 
meaning of $c_{r}$. It is the eigenvalue of the zero mode of the $\mathcal{W}_{\infty}$ spin-3 generator on the dual primary. In terms of $\mathrm{hs}[\lambda]$, this is the eigenvalue of $V_{0}^{3}$ on the highest weight. A straightforward calculation gives indeed, see appendix (D),

$$
V_{0}^{3}\left|\mathrm{hw}_{K}\right\rangle=\frac{K}{6}(\lambda+K)(\lambda+2 K)\left|\mathrm{hw}_{K}\right\rangle .
$$

We stress again that we are not attempting to prove the resummation (7.3) and we simply provide it as a suggestive compact conjecture to be demonstrated elsewhere.

\section{$7.2 \quad$ Next-to-leading order}

At the next order, we would like to capture the subleading terms $\rho_{n, 1}$ in (7.1). It turns out that this is possible by using the following modified formula

$$
R_{r} \stackrel{\lambda \rightarrow \infty}{=} \exp \left(\frac{f_{L O}}{\sin ^{2} \frac{\mathcal{Z}}{2}} \frac{c_{r} \alpha}{\tau^{2}}+K \frac{f_{N L O}}{\sin ^{4} \frac{\mathcal{Z}}{2}} \frac{\left(\lambda^{2} \alpha\right)^{2}}{\lambda \tau^{4}}\right)+\mathcal{O}\left(\alpha^{n} \lambda^{2 n-2}\right),
$$

where

$$
\begin{aligned}
f_{N L O}= & \frac{\mathcal{Z}^{2}}{16}+\frac{1}{16} \mathcal{Z}^{2} \cos \mathcal{Z}-\frac{5}{24} \mathcal{Z} \sin \mathcal{Z}-\frac{1}{48} \mathcal{Z} \sin (2 \mathcal{Z}) \\
& -\frac{\cos \mathcal{Z}}{12}-\frac{1}{24} \cos (2 \mathcal{Z})+\frac{1}{8} .
\end{aligned}
$$

Again, a single coefficient function $f_{N L O}$ is enough to capture many terms in (7.1). We remark that the term involving $f_{L O}$ also contributes to the determination of $\rho_{n, 1}$, but we did not find a sensible way of disentangling this mixing. The expression of the function $f_{N L O}$ has no simple explanation. A deeper understanding of it could come from a currently unavailable analytical proof of our resummation conjecture.

\subsection{Next-next-to-leading order}

Pushing further the computation, we would like to capture the subleading terms $\rho_{n, 2}$ in (7.1). It turns out that this is not possible by introducing a single further coefficient function. Nevertheless, two of them are enough. The explicit formula is

$$
\begin{aligned}
R_{r} \stackrel{\lambda \rightarrow \infty}{=} & \exp \left(\frac{f_{L O}}{\sin ^{2} \frac{\mathcal{Z}}{2}} \frac{c_{r} \alpha}{\tau^{2}}+K \frac{f_{N L O}}{\sin ^{4} \frac{\mathcal{Z}}{2}} \frac{\left(\lambda^{2} \alpha\right)^{2}}{\lambda \tau^{4}}\right. \\
& \left.+K^{2} \frac{f_{N^{2} L O, 1}}{\sin ^{4} \frac{\mathcal{Z}}{2}} \frac{\left(\lambda^{2} \alpha\right)^{2}}{\lambda^{2} \tau^{4}}+K \frac{f_{N^{2} L O, 2}}{\sin ^{6} \frac{\lambda^{2}}{2}} \frac{\left(\lambda^{2}\right.}{\lambda^{2} \tau^{6}}\right)+\mathcal{O}\left(\alpha^{n} \lambda^{2 n-3}\right)
\end{aligned}
$$

where

$$
\begin{aligned}
f_{N^{2} L O, 1}= & \frac{3 \mathcal{Z}^{2}}{8}+\frac{5}{16} \mathcal{Z}^{2} \cos \mathcal{Z}-\frac{29}{24} \mathcal{Z} \sin \mathcal{Z}-\frac{7 \cos \mathcal{Z}}{12} \\
& -\frac{19}{96} \cos (2 \mathcal{Z})-\frac{1}{6} \mathcal{Z} \sin \mathcal{Z} \cos \mathcal{Z}+\frac{25}{32}
\end{aligned}
$$




$$
\begin{aligned}
f_{N^{2} L O, 2}= & -\frac{3 \mathcal{Z}^{3}}{64}-\frac{5}{96} \mathcal{Z}^{3} \cos \mathcal{Z}-\frac{1}{192} \mathcal{Z}^{3} \cos (2 \mathcal{Z})+\frac{19}{96} \mathcal{Z}^{2} \sin \mathcal{Z}+\frac{11}{96} \mathcal{Z}^{2} \sin \mathcal{Z} \cos \mathcal{Z} \\
& -\frac{17 \mathcal{Z}}{96}+\frac{5 \sin \mathcal{Z}}{32}-\frac{1}{16} \sin (2 \mathcal{Z})-\frac{1}{96} \sin (3 \mathcal{Z}) \\
& +\frac{7}{192} \mathcal{Z} \cos \mathcal{Z}+\frac{13}{96} \mathcal{Z} \cos (2 \mathcal{Z})+\frac{1}{192} \mathcal{Z} \cos (3 \mathcal{Z}) .
\end{aligned}
$$

Conventionally, we kept the additional contributions in (7.8) in exponential form, but it is clear that more perturbative data should be welcome in order to assess its higher order validity. Anyway, it works at the order $\mathcal{O}\left(\alpha^{5}\right)$ included.

\subsection{Higher orders}

We explored the possibility of a resummation of the next terms $\mathcal{O}\left(\alpha^{n} \lambda^{2 n-3}\right)$. For all the three representation, this is possible by adding three new coefficient functions. The structure is now

$$
\begin{aligned}
R_{r} \stackrel{\lambda \rightarrow \infty}{=} & \exp \left(\frac{f_{L O}}{\sin ^{2} \frac{\mathcal{Z}}{2}} \frac{c_{r} \alpha}{\tau^{2}}+K \frac{f_{N L O}}{\sin ^{4} \frac{\mathcal{Z}}{2}} \frac{\left(\lambda^{2} \alpha\right)^{2}}{\lambda \tau^{4}}+K^{2} \frac{f_{N^{2} L O, 1}}{\sin ^{4} \frac{\mathcal{Z}}{2}} \frac{\left(\lambda^{2} \alpha\right)^{2}}{\lambda^{2} \tau^{4}}+K \frac{f_{N^{2} L O, 2}}{\sin ^{6} \frac{\mathcal{Z}}{2}} \frac{\left(\lambda^{2} \alpha\right)^{3}}{\lambda^{2} \tau^{6}}\right. \\
& \left.+\frac{f_{N^{3} L O, 1}^{(r)}}{\sin ^{4} \frac{\mathcal{Z}}{2}} \frac{\left(\lambda^{2} \alpha\right)^{2}}{\lambda^{3} \tau^{4}}+K^{2} \frac{f_{N^{3} L O, 2}}{\sin ^{6} \frac{\left(\lambda^{2} \alpha\right)^{3}}{2}} \frac{f_{N^{3} L O, 3}}{\lambda^{3} \tau^{6}} \frac{\left(\lambda^{2} \alpha\right)^{4}}{\sin ^{8} \frac{\mathcal{Z}}{2}}\right)+\mathcal{O}\left(\alpha^{n} \lambda^{2 n-4}\right),
\end{aligned}
$$

The functions $f_{N^{3} L O, 2-3}$ depend trivially on $K$ and are written in appendix (E). The function $f_{N^{3} L O, 1}^{(r)}$ has a more complicated dependence on $K$ and is reported also in appendix (E) for the three considered representations. What seems to be firmly established is that there are indeed intriguing hidden relations between the various functions $\rho_{n, m}(\mathcal{Z})$.

\subsection{Resummation structure in the zero temperature limit}

A further insight about the resummation issue comes from the analysis of the zero temperature limit in the Bergshoeff-Blencowe-Stelle limit. Let us begin with the fundamental representation. From (6.7), we are lead to consider the function

$$
\mathcal{R}_{\square}(\xi)=\sum_{n=0}^{\infty} \frac{\xi^{n}}{n !} \frac{\Gamma(2 n+\lambda+1)}{\Gamma(\lambda+1)} .
$$

Taking the logarithm and expanding in powers of $\lambda^{2} \xi$, we find

$$
\begin{aligned}
\log \mathcal{R}_{\square}(\xi)= & \lambda^{2} \xi\left(1+\frac{3}{\lambda}+\frac{2}{\lambda^{2}}\right)+\left(\lambda^{2} \xi\right)^{2}\left(\frac{2}{\lambda}+\frac{11}{\lambda^{2}}+\frac{19}{\lambda^{3}}+\frac{10}{\lambda^{4}}\right) \\
& +\left(\lambda^{2} \xi\right)^{3}\left(\frac{20}{3 \lambda^{2}}+\frac{56}{\lambda^{3}}+\frac{512}{3 \lambda^{4}}+\frac{220}{\lambda^{5}}+\frac{296}{3 \lambda^{6}}\right) \\
& +\left(\lambda^{2} \xi\right)^{4}\left(\frac{28}{\lambda^{3}}+\frac{326}{\lambda^{4}}+\frac{1492}{\lambda^{5}}+\frac{3320}{\lambda^{6}}+\frac{3538}{\lambda^{7}}+\frac{1412}{\lambda^{8}}\right) \\
& +\left(\lambda^{2} \xi\right)^{5}\left(\frac{672}{5 \lambda^{4}}+\frac{10208}{5 \lambda^{5}}+\frac{12832}{\lambda^{6}}+\frac{42400}{\lambda^{7}}+\frac{384464}{5 \lambda^{8}}+\frac{71536}{\lambda^{9}}+\frac{130592}{5 \lambda^{10}}\right) \\
& +\mathcal{O}\left(\xi^{6}\right) .
\end{aligned}
$$


The structure of this double expansion is interesting. The term $\sim\left(\lambda^{2} \xi\right)^{n}$ multiplies inverse powers $(1 / \lambda)^{m}$ with $n-1 \leq m \leq 2 n$. This means that if we want to capture all terms of the form $\left(\lambda^{2} \xi\right)^{n} / \lambda^{m}$, we need only to exponentiate a finite number of contributions. In more details, we can write $\mathcal{R}_{\square}(\xi)$ as

$$
\begin{aligned}
\mathcal{R}_{\square}(\xi)= & \exp \left[(\lambda+1)(\lambda+2) \xi+\frac{2}{\lambda}\left(\lambda^{2} \xi\right)^{2}+\frac{1}{\lambda^{2}}\left(11\left(\lambda^{2} \xi\right)^{2}+\frac{20}{3}\left(\lambda^{2} \xi\right)^{3}\right)\right. \\
& \left.+\frac{1}{\lambda^{3}}\left(19\left(\lambda^{2} \xi\right)^{2}+56\left(\lambda^{2} \xi\right)^{3}+28\left(\lambda^{2} \xi\right)^{4}\right)+\cdots\right] .
\end{aligned}
$$

The structure is the same as in (7.11) apart from the fact that the dependence on $\mathcal{Z}$, hidden inside $\xi$, is now trivial. We can also inspect the dependence on $K$ by considering the 2 box representation for which we have computed the zero temperature limit. A completely similar calculation gives indeed

$$
\begin{aligned}
\mathcal{R}_{\boxminus}(\xi)= & \exp \left[2(\lambda+2)(\lambda+4) \xi+\frac{2 \cdot 2}{\lambda}\left(\lambda^{2} \xi\right)^{2}+\frac{1}{\lambda^{2}}\left(2^{2} \cdot 11\left(\lambda^{2} \xi\right)^{2}+2 \cdot \frac{20}{3}\left(\lambda^{2} \xi\right)^{3}\right)\right. \\
& \left.+\frac{1}{\lambda^{3}}\left(146\left(\lambda^{2} \xi\right)^{2}+2^{2} \cdot 56\left(\lambda^{2} \xi\right)^{3}+2 \cdot 28\left(\lambda^{2} \xi\right)^{4}\right)+\cdots\right] .
\end{aligned}
$$

The coefficients are those in $\mathcal{R}_{\square}(\xi)$ after the same scaling by a power of $K=2$ precisely as in (7.11). The only exception is the term $\frac{1}{\lambda^{3}}\left(\lambda^{2} \xi\right)^{2}$ that, like $f_{N^{3} L O, 1}^{(r)}$ in (7.11) has a more complicated dependence on $K$.

\section{Conclusions}

In this paper, we have considered the duality between Vasiliev 3D higher spin gravity and $\mathcal{W}_{N}$ minimal models. We have computed the correlator of the bulk complex scalar in the background of a higher spin black hole and we have determined the exact dependence on the symmetry parameter $\lambda$ at order $\mathcal{O}\left(\alpha^{5}\right)$ where $\alpha$ is the chemical potential associated with the spin-3 charge of the black hole. We have analysed the perturbative data in the large $\lambda$ Bergshoeff-Blencowe-Stelle limit where we had some expectations that some simplification could occur. We found special regularities and proposed a conjectured resummation of all the leading and subleading contributions that take the form $\mathcal{O}\left(\alpha^{n} \cdot\left\{\lambda^{2 n}, \lambda^{2 n-p}\right\}\right)$, with $p=0,1,2,3$.

We believe that such non-trivial property of the perturbative expansion could be explained, and possibly extended, in terms of a systematic $1 / \mathcal{N}$ expansion of the $\mathfrak{s t}(\mathcal{N}) \oplus \mathfrak{s t}(\mathcal{N})$ Chern-Simons gravity theory. Alternatively, a suitable contraction of the symmetry algebra hs $[\lambda]$ could be devised in order to capture the corrections considered in this paper. Work is in progress on this subject. 


\section{A The infinite dimensional algebra hs $[\lambda]$}

The hs $[\lambda]$ algebra is generated by $V_{m}^{s}$ with $s \geq 2$ and $|m|<s$. The lone-star operation [19] is the associative product defined by

$$
V_{m}^{s} \star V_{n}^{t}=\frac{1}{2} \sum_{u=1}^{s+t-1} g_{u}^{s t}(m, n, \lambda) V_{m+n}^{s+t-u},
$$

with

$$
\begin{aligned}
& g_{u}^{s t}(m, n, \lambda)=\frac{(1 / 4)^{u-2}}{2(u-1) !}{ }^{4} F_{3}\left(\begin{array}{c}
\frac{1}{2}+\lambda, \frac{1}{2}-\lambda, \frac{2-u}{2}, \frac{1-u}{2} \\
\frac{3}{2}-s, \frac{3}{2}-t, \frac{1}{2}+s+t-u
\end{array} \mid 1\right) \times \\
& \sum_{k=0}^{u-1}(-1)^{k}\left(\begin{array}{c}
u-1 \\
k
\end{array}\right)(s-1+m)_{u-1-k}^{-}(s-1-m)_{k}^{-}(t-1+n)_{k}^{-}(t-1-n)_{u-1-k}^{-},
\end{aligned}
$$

where $(a)_{n}^{-}=a(a-1) \cdots(a-n+1)$ is the descending Pochammer symbol. An infinitedimensional matrix representation of $\mathrm{hs}[\lambda]$ is obtained by starting with the following infinite-dimensional matrix representation of $\mathfrak{s l}(2)$ (we list the non-zero elements )

$$
\begin{aligned}
\left(V_{0}^{2}\right)_{n, n} & =\frac{-\lambda+1}{2}-n, \\
\left(V_{1}^{2}\right)_{n+1, n} & =-\sqrt{(-\lambda-n) n}, \\
\left(V_{-1}^{2}\right)_{n, n+1} & =\sqrt{(-\lambda-n) n},
\end{aligned}
$$

and building the other generators according to

$$
V_{m}^{s}=(-1)^{s-1-m} \frac{(s+m-1) !}{(2 s-2) !} \operatorname{Ad}_{V_{-1}^{2}}^{s-m-1}\left(V_{1}^{2}\right)^{s-1} .
$$

A very important property of this representation is that setting $\lambda=-\mathcal{N}$, a negative integer, and restricting $V_{m}^{s}$ to the set with $s \leq \mathcal{N}$, we obtain a $\mathcal{N} \times \mathcal{N}$ dimensional representation of $\mathfrak{s l}(\mathcal{N})$ by restricting the infinite dimensional matrices to their first $\mathcal{N}$ rows and columns. Also, the lone-star product becomes simply matrix multiplication.

\section{B Non-zero polynomials $p_{m, k}^{(n)}(\lambda)$ for $n \leq 4$}

In this section we report the explicit expressions for the polynomials appearing in (4.3).

\section{B.1 Fundamental representation}

Let us define

$$
f=(\lambda+1)(\lambda+2) \text {. }
$$

We have:

$$
\begin{aligned}
p_{0,1}^{(1)}(\lambda) & =\frac{f}{4} \\
p_{1,0}^{(1)}(\lambda) & =-\frac{f}{6} \\
p_{1,1}^{(1)}(\lambda) & =-\frac{f}{12}
\end{aligned}
$$




$$
\begin{aligned}
& p_{0,0}^{(2)}(\lambda)=\frac{f}{64}(\lambda+4)(\lambda+7) \\
& p_{0,1}^{(2)}(\lambda)=-\frac{f}{12}(\lambda+4) \\
& p_{0,2}^{(2)}(\lambda)=-\frac{f}{192}(\lambda+4)(3 \lambda+5) \\
& p_{1,1}^{(2)}(\lambda)=-\frac{f}{24}(\lambda+4)^{2} \\
& p_{1,2}^{(2)}(\lambda)=-\frac{f}{96}(\lambda+1)(\lambda+4) \\
& p_{2,0}^{(2)}(\lambda)=\frac{f}{64}\left(\lambda^{2}+7 \lambda+14\right) \\
& p_{2,1}^{(2)}(\lambda)=\frac{f}{144}(\lambda+2)(2 \lambda+11) \\
& p_{2,2}^{(2)}(\lambda)=\frac{f}{576}(\lambda+1)(\lambda+2) \\
& p_{0,1}^{(3)}(\lambda)=\frac{f}{1536}(\lambda+3)(\lambda+4)(\lambda+6)(3 \lambda+35) \\
& p_{0,2}^{(3)}(\lambda)=-\frac{f}{96}(\lambda+3)(\lambda+4)(\lambda+6) \\
& p_{0,3}^{(3)}(\lambda)=-\frac{f}{1536}(\lambda+1)(\lambda+3)(\lambda+4)(\lambda+6) \\
& p_{1,0}^{(3)}(\lambda)=-\frac{f}{1152}\left(3 \lambda^{4}+68 \lambda^{3}+539 \lambda^{2}+1942 \lambda+2488\right) \\
& p_{1,1}^{(3)}(\lambda)=-\frac{f}{1536}\left(\lambda^{4}+2 \lambda^{3}-89 \lambda^{2}-842 \lambda-1592\right) \\
& p_{1,2}^{(3)}(\lambda)=\frac{f}{384}\left(\lambda^{4}+20 \lambda^{3}+145 \lambda^{2}+418 \lambda+424\right) \\
& p_{1,3}^{(3)}(\lambda)=\frac{f}{4608}\left(3 \lambda^{4}+38 \lambda^{3}+149 \lambda^{2}+226 \lambda+88\right) \\
& p_{2,1}^{(3)}(\lambda)=\frac{f}{4608}(\lambda+6)\left(17 \lambda^{3}+236 \lambda^{2}+1093 \lambda+1594\right) \\
& p_{2,2}^{(3)}(\lambda)=\frac{f}{1152}(\lambda+2)(\lambda+6)(\lambda+7)(2 \lambda+5) \\
& p_{2,3}^{(3)}(\lambda)=\frac{f^{2}}{4608}(\lambda+2)(\lambda+6) \\
& p_{3,0}^{(3)}(\lambda)=-\frac{f}{10368}\left(11 \lambda^{4}+201 \lambda^{3}+1412 \lambda^{2}+4344 \lambda+4904\right) \\
& p_{3,1}^{(3)}(\lambda)=-\frac{f}{13824}\left(17 \lambda^{4}+318 \lambda^{3}+2093 \lambda^{2}+5892 \lambda+5972\right) \\
& p_{3,2}^{(3)}(\lambda)=-\frac{f}{3456}(\lambda+2)^{2}(\lambda+4)(\lambda+7) \\
& p_{3,3}^{(3)}(\lambda)=-\frac{f^{3}}{41472}
\end{aligned}
$$




$$
\begin{aligned}
& p_{0,0}^{(4)}(\lambda)=\frac{f}{147456}(\lambda+8)\left(9 \lambda^{5}+345 \lambda^{4}+4925 \lambda^{3}+32135 \lambda^{2}+100546 \lambda+117400\right) \\
& p_{0,1}^{(4)}(\lambda)=-\frac{f}{4608}(\lambda+8)\left(3 \lambda^{4}+82 \lambda^{3}+721 \lambda^{2}+2762 \lambda+3656\right) \\
& p_{0,2}^{(4)}(\lambda)=-\frac{f}{36864}(\lambda+8)\left(3 \lambda^{5}+99 \lambda^{4}+1055 \lambda^{3}+4925 \lambda^{2}+9526 \lambda+5896\right) \\
& p_{0,3}^{(4)}(\lambda)=\frac{f}{4608}(\lambda+8)\left(3 \lambda^{4}+50 \lambda^{3}+305 \lambda^{2}+778 \lambda+712\right) \\
& p_{0,4}^{(4)}(\lambda)=\frac{f}{147456}(\lambda+8)\left(3 \lambda^{5}+51 \lambda^{4}+319 \lambda^{3}+877 \lambda^{2}+1046 \lambda+392\right) \\
& p_{1,1}^{(4)}(\lambda)=-\frac{f}{9216}\left(3 \lambda^{6}+124 \lambda^{5}+2013 \lambda^{4}+16904 \lambda^{3}+76708 \lambda^{2}+177768 \lambda+163952\right) \\
& p_{1,2}^{(4)}(\lambda)=-\frac{f}{18432}\left(\lambda^{6}+7 \lambda^{5}-287 \lambda^{4}-5551 \lambda^{3}-37282 \lambda^{2}-109368 \lambda-117664\right) \\
& p_{1,3}^{(4)}(\lambda)=\frac{f}{9216}(\lambda+2)\left(\lambda^{5}+34 \lambda^{4}+443 \lambda^{3}+2642 \lambda^{2}+7368 \lambda+7656\right) \\
& p_{1,4}^{(4)}(\lambda)=\frac{f}{36864}(\lambda+1)\left(\lambda^{5}+22 \lambda^{4}+171 \lambda^{3}+598 \lambda^{2}+872 \lambda+352\right) \\
& p_{2,0}^{(4)}(\lambda)=\frac{f}{221184}\left(51 \lambda^{6}+1955 \lambda^{5}+30137 \lambda^{4}+245921 \lambda^{3}+1101812 \lambda^{2}+2564444 \lambda+2406080\right) \\
& p_{2,1}^{(4)}(\lambda)=\frac{f}{55296}\left(6 \lambda^{6}+169 \lambda^{5}+1870 \lambda^{4}+7807 \lambda^{3}+1672 \lambda^{2}-73748 \lambda-137360\right) \\
& p_{2,2}^{(4)}(\lambda)=-\frac{f}{27648}\left(6 \lambda^{6}+229 \lambda^{5}+3541 \lambda^{4}+27937 \lambda^{3}+118309 \lambda^{2}+253066 \lambda+213808\right) \\
& p_{2,3}^{(4)}(\lambda)=-\frac{f}{55296}(\lambda+2)\left(6 \lambda^{5}+173 \lambda^{4}+1876 \lambda^{3}+9351 \lambda^{2}+21338 \lambda+18232\right) \\
& p_{2,4}^{(4)}(\lambda)=-\frac{f^{2}}{221184}\left(3 \lambda^{4}+50 \lambda^{3}+245 \lambda^{2}+406 \lambda+160\right) \\
& p_{3,1}^{(4)}(\lambda)=-\frac{f}{82944}(\lambda+8)\left(19 \lambda^{5}+529 \lambda^{4}+5869 \lambda^{3}+31759 \lambda^{2}+82816 \lambda+82876\right) \\
& p_{3,2}^{(4)}(\lambda)=-\frac{f}{165888}(\lambda+8)\left(25 \lambda^{5}+643 \lambda^{4}+6199 \lambda^{3}+28093 \lambda^{2}+60556 \lambda+50140\right) \\
& p_{3,3}^{(4)}(\lambda)=-\frac{f}{27648}(\lambda+2)^{2}(\lambda+8)\left(\lambda^{3}+15 \lambda^{2}+63 \lambda+85\right) \\
& p_{3,4}^{(4)}(\lambda)=-\frac{f^{3}}{331776}(\lambda+1)(\lambda+8) \\
& p_{4,0}^{(4)}(\lambda)=\frac{f}{3981312}\left(227 \lambda^{6}+7659 \lambda^{5}+107715 \lambda^{4}+795357 \lambda^{3}+3235542 \lambda^{2}+6848460 \lambda+5873560\right) \\
& p_{4,1}^{(4)}(\lambda)=\frac{f}{497664}\left(38 \lambda^{6}+1287 \lambda^{5}+17562 \lambda^{4}+124461 \lambda^{3}+482406 \lambda^{2}+968940 \lambda+787624\right) \\
& p_{4,2}^{(4)}(\lambda)=\frac{f}{995328}\left(25 \lambda^{6}+765 \lambda^{5}+9249 \lambda^{4}+56655 \lambda^{3}+187086 \lambda^{2}+317556 \lambda+217496\right) \\
& p_{4,3}^{(4)}(\lambda)=\frac{f}{497664}(\lambda+2)^{3}\left(2 \lambda^{3}+33 \lambda^{2}+168 \lambda+299\right) \\
& p_{4,4}^{(4)}(\lambda)=\frac{f^{4}}{3981312}
\end{aligned}
$$




\section{B.2 2-Antisymmetric representation}

Let us define

$$
f=(\lambda+2)(\lambda+4) .
$$

We have:

$$
\begin{aligned}
& p_{0,1}^{(1)}(\lambda)=\frac{f}{2} \\
& p_{1,0}^{(1)}(\lambda)=-\frac{f}{3} \\
& p_{1,1}^{(1)}(\lambda)=-\frac{f}{6} \\
& p_{0,0}^{(2)}(\lambda)=\frac{1}{16}(\lambda+2)\left(\lambda^{3}+14 \lambda^{2}+74 \lambda+121\right) \\
& p_{0,1}^{(2)}(\lambda)=-\frac{1}{6}(\lambda+2)\left(\lambda^{2}+12 \lambda+26\right) \\
& p_{0,2}^{(2)}(\lambda)=-\frac{1}{48}(\lambda+2)(\lambda+5)\left(3 \lambda^{2}+19 \lambda+31\right) \\
& p_{1,1}^{(2)}(\lambda)=-\frac{1}{12}(\lambda+2)\left(2 \lambda^{3}+25 \lambda^{2}+112 \lambda+164\right) \\
& p_{1,2}^{(2)}(\lambda)=-\frac{1}{24}(\lambda+2)\left(\lambda^{3}+11 \lambda^{2}+38 \lambda+43\right) \\
& p_{2,0}^{(2)}(\lambda)=\frac{1}{16}(\lambda+2)\left(\lambda^{3}+12 \lambda^{2}+52 \lambda+74\right) \\
& p_{2,1}^{(2)}(\lambda)=\frac{f}{72}(\lambda+4)(4 \lambda+17) \\
& p_{2,2}^{(2)}(\lambda)=\frac{f^{2}}{144} \\
& p_{0,1}^{(3)}(\lambda)=\frac{f}{192}(\lambda+3)(\lambda+6)\left(3 \lambda^{2}+37 \lambda+167\right) \\
& p_{0,2}^{(3)}(\lambda)=-\frac{f}{24}(\lambda+3)(\lambda+6)(\lambda+8) \\
& p_{0,3}^{(3)}(\lambda)=-\frac{f}{192}(\lambda+3)(\lambda+6)\left(\lambda^{2}+7 \lambda+13\right) \\
& p_{1,0}^{(3)}(\lambda)=-\frac{f}{144}\left(3 \lambda^{4}+61 \lambda^{3}+503 \lambda^{2}+1979 \lambda+2794\right) \\
& p_{1,1}^{(3)}(\lambda)=-\frac{f}{192}\left(\lambda^{4}+10 \lambda^{3}-8 \lambda^{2}-457 \lambda-1070\right) \\
& p_{1,2}^{(3)}(\lambda)=\frac{f}{48}\left(\lambda^{4}+19 \lambda^{3}+145 \lambda^{2}+497 \lambda+622\right) \\
& p_{1,3}^{(3)}(\lambda)=\frac{f}{576}\left(3 \lambda^{4}+46 \lambda^{3}+248 \lambda^{2}+581 \lambda+502\right) \\
& p_{2,1}^{(3)}(\lambda)=\frac{f}{576}\left(17 \lambda^{4}+322 \lambda^{3}+2488 \lambda^{2}+9026 \lambda+11988\right) \\
& p_{2,2}^{(3)}(\lambda)=\frac{f}{288}(\lambda+5)\left(4 \lambda^{3}+51 \lambda^{2}+224 \lambda+324\right)
\end{aligned}
$$

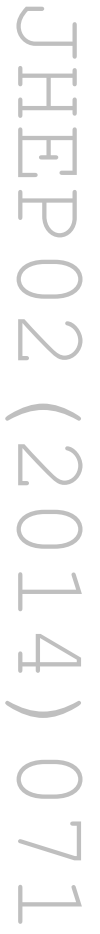




$$
\begin{aligned}
& p_{2,3}^{(3)}(\lambda)=\frac{f}{576}(\lambda+2)\left(\lambda^{3}+12 \lambda^{2}+44 \lambda+54\right) \\
& p_{3,0}^{(3)}(\lambda)=-\frac{f\left(22 \lambda^{4}+399 \lambda^{3}+2953 \lambda^{2}+10212 \lambda+13072\right)}{2592} \\
& p_{3,1}^{(3)}(\lambda)=-\frac{f\left(17 \lambda^{4}+312 \lambda^{3}+2216 \lambda^{2}+7284 \lambda+8936\right)}{1728} \\
& p_{3,2}^{(3)}(\lambda)=-\frac{1}{864} f(\lambda+4)^{2}(\lambda+5)(2 \lambda+7) \\
& p_{3,3}^{(3)}(\lambda)=-\frac{f^{3}}{5184}
\end{aligned}
$$

$$
\begin{aligned}
& p_{0,0}^{(4)}(\lambda)=\frac{(\lambda+2)\left(9 \lambda^{7}+366 \lambda^{6}+6632 \lambda^{5}+68870 \lambda^{4}+439976 \lambda^{3}+1700624 \lambda^{2}+3588743 \lambda+3127340\right)}{9216} \\
& p_{0,1}^{(4)}(\lambda)=-\frac{1}{576}(\lambda+2)\left(3 \lambda^{6}+110 \lambda^{5}+1712 \lambda^{4}+14507 \lambda^{3}+68774 \lambda^{2}+167078 \lambda+159968\right) \\
& p_{0,2}^{(4)}(\lambda)=-\frac{(\lambda+2)\left(3 \lambda^{7}+114 \lambda^{6}+1880 \lambda^{5}+17234 \lambda^{4}+93488 \lambda^{3}+296192 \lambda^{2}+505949 \lambda+360356\right)}{2304} \\
& p_{0,3}^{(4)}(\lambda)=\frac{1}{576}(\lambda+2)\left(3 \lambda^{6}+94 \lambda^{5}+1216 \lambda^{4}+8299 \lambda^{3}+31366 \lambda^{2}+62182 \lambda+50464\right) \\
& p_{0,4}^{(4)}(\lambda)=\frac{(\lambda+2)\left(3 \lambda^{7}+90 \lambda^{6}+1144 \lambda^{5}+8002 \lambda^{4}+33304 \lambda^{3}+82672 \lambda^{2}+113389 \lambda+66148\right)}{9216} \\
& p_{1,1}^{(4)}(\lambda)=-\frac{(\lambda+2)(\lambda+8)\left(6 \lambda^{6}+181 \lambda^{5}+2414 \lambda^{4}+18004 \lambda^{3}+77105 \lambda^{2}+174640 \lambda+160172\right)}{1152} \\
& p_{1,2}^{(4)}(\lambda)=-\frac{(\lambda+2)\left(\lambda^{7}+21 \lambda^{6}+78 \lambda^{5}-1894 \lambda^{4}-26049 \lambda^{3}-139840 \lambda^{2}-349987 \lambda-335548\right)}{1152} \\
& p_{1,3}^{(4)}(\lambda)=\frac{(\lambda+2)\left(2 \lambda^{7}+71 \lambda^{6}+1106 \lambda^{5}+9644 \lambda^{4}+50411 \lambda^{3}+156968 \lambda^{2}+268548 \lambda+194208\right)}{1152} \\
& p_{1,4}^{(4)}(\lambda)=\frac{(\lambda+2)\left(\lambda^{7}+29 \lambda^{6}+350 \lambda^{5}+2298 \lambda^{4}+8903 \lambda^{3}+20448 \lambda^{2}+25837 \lambda+13828\right)}{2304} \\
& p_{2,0}^{(4)}(\lambda)=\frac{(\lambda+2)\left(51 \lambda^{7}+1870 \lambda^{6}+30420 \lambda^{5}+285351 \lambda^{4}+1653324 \lambda^{3}+5831100 \lambda^{2}+11379570 \lambda+9326444\right)}{13824} \\
& p_{2,1}^{(4)}(\lambda)=\frac{(\lambda+2)\left(12 \lambda^{7}+379 \lambda^{6}+5118 \lambda^{5}+36678 \lambda^{4}+142599 \lambda^{3}+260448 \lambda^{2}+86208 \lambda-213256\right)}{6912} \\
& p_{2,2}^{(4)}(\lambda)=-\frac{(\lambda+2)\left(12 \lambda^{7}+439 \lambda^{6}+7134 \lambda^{5}+66189 \lambda^{4}+375123 \lambda^{3}+1281228 \lambda^{2}+2410755 \lambda+1907840\right)}{3456} \\
& p_{2,3}^{(4)}(\lambda)=-\frac{(\lambda+2)\left(12 \lambda^{7}+395 \lambda^{6}+5598 \lambda^{5}+44166 \lambda^{4}+208983 \lambda^{3}+592416 \lambda^{2}+930144 \lambda+622456\right)}{6912} \\
& p_{2,4}^{(4)}(\lambda)=-\frac{(\lambda+2)^{2}\left(\lambda^{3}+14 \lambda^{2}+56 \lambda+70\right)\left(3 \lambda^{3}+34 \lambda^{2}+128 \lambda+169\right)}{13824} \\
& p_{3,1}^{(4)}(\lambda)=-\frac{f\left(38 \lambda^{6}+1194 \lambda^{5}+16485 \lambda^{4}+127347 \lambda^{3}+574173 \lambda^{2}+1399308 \lambda+1395856\right)}{10368} \\
& p_{3,2}^{(4)}(\lambda)=-\frac{f\left(25 \lambda^{6}+759 \lambda^{5}+9822 \lambda^{4}+69519 \lambda^{3}+282600 \lambda^{2}+618708 \lambda+560402\right)}{10368} \\
& p_{3,3}^{(4)}(\lambda)=-\frac{f(\lambda+4)\left(2 \lambda^{5}+46 \lambda^{4}+419 \lambda^{3}+1897 \lambda^{2}+4295 \lambda+3880\right)}{3456} \\
& p_{3,4}^{(4)}(\lambda)=-\frac{f^{2}(\lambda+2)\left(\lambda^{3}+13 \lambda^{2}+50 \lambda+65\right)}{20736} \\
& p_{4,0}^{(4)}(\lambda)= \\
& (\lambda+2)\left(227 \lambda^{7}+7802 \lambda^{6}+119208 \lambda^{5}+1045128 \lambda^{4}+5645904 \lambda^{3}+18594528 \lambda^{2}+34117144 \lambda+26560720\right)
\end{aligned}
$$




$$
\begin{aligned}
& p_{4,1}^{(4)}(\lambda)=\frac{(\lambda+2)\left(152 \lambda^{7}+5234 \lambda^{6}+78852 \lambda^{5}+676263 \lambda^{4}+3558120 \lambda^{3}+11403312 \lambda^{2}+20392768 \lambda+15518560\right)}{124416} \\
& p_{4,2}^{(4)}(\lambda)=\frac{(\lambda+2)\left(25 \lambda^{7}+820 \lambda^{6}+11607 \lambda^{5}+92220 \lambda^{4}+444648 \lambda^{3}+1298208 \lambda^{2}+2115848 \lambda+1476692\right)}{62208} \\
& p_{4,3}^{(4)}(\lambda)=\frac{f(\lambda+4)^{3}\left(8 \lambda^{3}+102 \lambda^{2}+420 \lambda+577\right)}{124416} \\
& p_{4,4}^{(4)}(\lambda)=\frac{f^{4}}{248832}
\end{aligned}
$$

\section{B.3 3-Antisymmetric representation}

Let us define

$$
f=(\lambda+2)(\lambda+4) .
$$

We have:

$$
\begin{aligned}
& p_{0,1}^{(1)}(\lambda)=\frac{3 f}{4} \\
& p_{1,0}^{(1)}(\lambda)=-\frac{f}{2} \\
& p_{1,1}^{(1)}(\lambda)=-\frac{f}{4} \\
& p_{0,0}^{(2)}(\lambda)=\frac{3}{64}(\lambda+3)(\lambda+4)\left(3 \lambda^{2}+41 \lambda+178\right) \\
& p_{0,1}^{(2)}(\lambda)=-\frac{1}{4}(\lambda+3)(\lambda+4)(\lambda+14) \\
& p_{0,2}^{(2)}(\lambda)=-\frac{1}{64}(\lambda+3)(\lambda+4)(\lambda+5)(9 \lambda+62) \\
& p_{1,1}^{(2)}(\lambda)=-\frac{1}{8}(\lambda+3)(\lambda+4)\left(3 \lambda^{2}+38 \lambda+136\right) \\
& p_{1,2}^{(2)}(\lambda)=-\frac{1}{32}(\lambda+3)(\lambda+4)\left(3 \lambda^{2}+35 \lambda+94\right) \\
& p_{2,0}^{(2)}(\lambda)=\frac{3}{64}(\lambda+3)\left(3 \lambda^{3}+49 \lambda^{2}+276 \lambda+508\right) \\
& p_{2,1}^{(2)}(\lambda)=\frac{f}{16}(\lambda+6)(2 \lambda+9) \\
& p_{2,2}^{(2)}(\lambda)=\frac{f^{2}}{64} \\
& p_{0,1}^{(3)}(\lambda)=\frac{3 f}{512}(\lambda+4)\left(9 \lambda^{3}+182 \lambda^{2}+1419 \lambda+3822\right) \\
& p_{0,2}^{(3)}(\lambda)=-\frac{3 f}{32}(\lambda+4)\left(\lambda^{2}+19 \lambda+74\right) \\
& p_{0,3}^{(3)}(\lambda)=-\frac{f}{512}(\lambda+4)\left(9 \lambda^{3}+150 \lambda^{2}+811 \lambda+1454\right) \\
& p_{1,0}^{(3)}(\lambda)=-\frac{f}{128}\left(9 \lambda^{4}+212 \lambda^{3}+2009 \lambda^{2}+8758 \lambda+14072\right) \\
& p_{1,1}^{(3)}(\lambda)=-\frac{f}{512}\left(9 \lambda^{4}+150 \lambda^{3}+583 \lambda^{2}-1662 \lambda-8200\right)
\end{aligned}
$$




$$
\begin{aligned}
& p_{1,2}^{(3)}(\lambda)=\frac{f}{128}\left(9 \lambda^{4}+204 \lambda^{3}+1825 \lambda^{2}+7302 \lambda+10808\right) \\
& p_{1,3}^{(3)}(\lambda)=\frac{f}{512}\left(9 \lambda^{4}+182 \lambda^{3}+1319 \lambda^{2}+4162 \lambda+4856\right) \\
& p_{2,1}^{(3)}(\lambda)=\frac{f}{512}\left(51 \lambda^{4}+1154 \lambda^{3}+10399 \lambda^{2}+42712 \lambda+65204\right) \\
& p_{2,2}^{(3)}(\lambda)=\frac{f}{128}(\lambda+5)\left(6 \lambda^{3}+101 \lambda^{2}+576 \lambda+1084\right) \\
& p_{2,3}^{(3)}(\lambda)=\frac{f}{512}(\lambda+3)\left(3 \lambda^{3}+49 \lambda^{2}+252 \lambda+428\right) \\
& p_{3,0}^{(3)}(\lambda)=-\frac{f}{384}\left(11 \lambda^{4}+243 \lambda^{3}+2124 \lambda^{2}+8424 \lambda+12456\right) \\
& p_{3,1}^{(3)}(\lambda)=-\frac{f}{512}\left(17 \lambda^{4}+378 \lambda^{3}+3221 \lambda^{2}+12396 \lambda+17876\right) \\
& p_{3,2}^{(3)}(\lambda)=-\frac{f}{128}(\lambda+4)(\lambda+5)(\lambda+6)^{2} \\
& p_{3,3}^{(3)}(\lambda)=-\frac{f^{3}}{1536}
\end{aligned}
$$

$$
\begin{aligned}
& p_{0,0}^{(4)}(\lambda)= \\
& \underline{(\lambda+3)\left(243 \lambda^{7}+11043 \lambda^{6}+224709 \lambda^{5}+2623025 \lambda^{4}+18820392 \lambda^{3}+82210172 \lambda^{2}+199702896 \lambda+205364480\right)} \\
& 49152 \\
& p_{0,1}^{(4)}(\lambda)=-\frac{(\lambda+3)\left(27 \lambda^{6}+1200 \lambda^{5}+22213 \lambda^{4}+221052 \lambda^{3}+1232956 \lambda^{2}+3587088 \lambda+4202752\right)}{1536} \\
& p_{0,2}^{(4)}(\lambda)=-\frac{(\lambda+3)\left(81 \lambda^{7}+3537 \lambda^{6}+68055 \lambda^{5}+736907 \lambda^{4}+4786296 \lambda^{3}+18477716 \lambda^{2}+39096528 \lambda+34934528\right)}{12288} \\
& p_{0,3}^{(4)}(\lambda)=\frac{(\lambda+3)\left(27 \lambda^{6}+1104 \lambda^{5}+18149 \lambda^{4}+155004 \lambda^{3}+729404 \lambda^{2}+1799952 \lambda+1822976\right)}{1536} \\
& p_{0,4}^{(4)}(\lambda)=\frac{(\lambda+3)\left(81 \lambda^{7}+3105 \lambda^{6}+50583 \lambda^{5}+454651 \lambda^{4}+2438328 \lambda^{3}+7814356 \lambda^{2}+13871568 \lambda+10526464\right)}{49152} \\
& p_{1,1}^{(4)}(\lambda)=-\frac{(\lambda+3)\left(81 \lambda^{7}+3546 \lambda^{6}+68847 \lambda^{5}+763034 \lambda^{4}+5173692 \lambda^{3}+21283280 \lambda^{2}+48737472 \lambda+47501024\right)}{3072} \\
& p_{1,2}^{(4)}(\lambda)=-\frac{(\lambda+3)\left(27 \lambda^{7}+873 \lambda^{6}+9981 \lambda^{5}+27563 \lambda^{4}-381408 \lambda^{3}-3791260 \lambda^{2}-13237104 \lambda-16671424\right)}{6144} \\
& p_{1,3}^{(4)}(\lambda)=\frac{(\lambda+3)\left(9 \lambda^{7}+378 \lambda^{6}+6967 \lambda^{5}+72282 \lambda^{4}+452252 \lambda^{3}+1697424 \lambda^{2}+3527616 \lambda+3124704\right)}{1024} \\
& p_{1,4}^{(4)}(\lambda)=\frac{(\lambda+3)\left(27 \lambda^{7}+1017 \lambda^{6}+16125 \lambda^{5}+140059 \lambda^{4}+722016 \lambda^{3}+2215204 \lambda^{2}+3751824 \lambda+2707264\right)}{12288} \\
& p_{2,0}^{(4)}(\lambda)= \\
& \frac{(\lambda+3)\left(459 \lambda^{7}+19635 \lambda^{6}+371775 \lambda^{5}+4021241 \lambda^{4}+26675766 \lambda^{3}+107760380 \lambda^{2}+243267960 \lambda+234536384\right)}{24576} \\
& p_{2,1}^{(4)}(\lambda)=\frac{(\lambda+3)\left(18 \lambda^{7}+709 \lambda^{6}+11952 \lambda^{5}+110323 \lambda^{4}+592866 \lambda^{3}+1814348 \lambda^{2}+2841112 \lambda+1661984\right)}{2048} \\
& p_{2,2}^{(4)}(\lambda)=-\frac{(\lambda+3)\left(18 \lambda^{7}+769 \lambda^{6}+14541 \lambda^{5}+156459 \lambda^{4}+1026717 \lambda^{3}+4079068 \lambda^{2}+9022540 \lambda+8515840\right)}{1024} \\
& p_{2,3}^{(4)}(\lambda)=-\frac{(\lambda+3)\left(54 \lambda^{7}+2175 \lambda^{6}+37776 \lambda^{5}+365993 \lambda^{4}+2131686 \lambda^{3}+7453988 \lambda^{2}+14474568 \lambda+12027488\right)}{6144} \\
& p_{2,4}^{(4)}(\lambda)=-\frac{(\lambda+3)^{2}\left(9 \lambda^{6}+302 \lambda^{5}+4131 \lambda^{4}+29650 \lambda^{3}+118452 \lambda^{2}+250968 \lambda+221120\right)}{8192}
\end{aligned}
$$




$$
\begin{aligned}
& p_{3,1}^{(4)}(\lambda)=-\frac{(\lambda+3)\left(19 \lambda^{7}+797 \lambda^{6}+14783 \lambda^{5}+156299 \lambda^{4}+1011478 \lambda^{3}+3981140 \lambda^{2}+8760984 \lambda+8252288\right)}{1024} \\
& p_{3,2}^{(4)}(\lambda)=-\frac{(\lambda+3)\left(25 \lambda^{7}+1031 \lambda^{6}+18493 \lambda^{5}+186753 \lambda^{4}+1144046 \lambda^{3}+4240252 \lambda^{2}+8776344 \lambda+7795072\right)}{2048} \\
& p_{3,3}^{(4)}(\lambda)=-\frac{f(\lambda+6)\left(3 \lambda^{5}+81 \lambda^{4}+871 \lambda^{3}+4667 \lambda^{2}+12470 \lambda+13280\right)}{1024} \\
& p_{3,4}^{(4)}(\lambda)=-\frac{f^{2}(\lambda+3)\left(\lambda^{3}+17 \lambda^{2}+90 \lambda+160\right)}{4096} \\
& p_{4,0}^{(4)}(\lambda)= \\
& \frac{(\lambda+3)\left(227 \lambda^{7}+9363 \lambda^{6}+169953 \lambda^{5}+1752177 \lambda^{4}+11034384 \lambda^{3}+42243432 \lambda^{2}+90519984 \lambda+83227920\right)}{49152} \\
& p_{4,1}^{(4)}(\lambda)=\frac{(\lambda+3)\left(38 \lambda^{7}+1569 \lambda^{6}+28248 \lambda^{5}+287283 \lambda^{4}+1778880 \lambda^{3}+6687912 \lambda^{2}+14078592 \lambda+12733680\right)}{6144} \\
& p_{4,2}^{(4)}(\lambda)=\frac{(\lambda+3)\left(25 \lambda^{7}+1005 \lambda^{6}+17427 \lambda^{5}+169035 \lambda^{4}+990348 \lambda^{3}+3502032 \lambda^{2}+6910848 \lambda+5858448\right)}{12288} \\
& p_{4,3}^{(4)}(\lambda)=\frac{f(\lambda+6)^{3}\left(2 \lambda^{3}+27 \lambda^{2}+120 \lambda+177\right)}{6144} \\
& p_{4,4}^{(4)}(\lambda)=\frac{f^{4}}{49152}
\end{aligned}
$$

\section{Zero temperature limit for the 2-antisymmetric representation}

In the 2-antisymmetric representation, the zero temperature limit of the scalar propagator is given by:

$$
\Phi=\lim _{\substack{\tau, \bar{\tau} \rightarrow \infty \\ \text { fixed } \mu}} e^{\Delta \rho}\left(\frac{1}{2}\langle 1|\mathcal{O}| 1\rangle\langle 2|\mathcal{O}| 2\rangle-\frac{1}{2}\langle 1|\mathcal{O}| 2\rangle\langle 2|\mathcal{O}| 1\rangle\right),
$$

where $\Delta$ is the conformal dimension of the dual scalar operator, related to the bulk scalar mass by $m^{2}=\Delta(\Delta-2)$, and $\mathcal{O}$ is the operator

$$
\mathcal{O}=e^{e^{\rho} \bar{z} V_{-1}^{2}} e^{-e^{\rho} z V_{1}^{2}} e^{\mu e^{2 \rho} \bar{z} V_{2}^{3}} .
$$

Using the fact that $V_{2}^{3}=V_{1}^{2} \star V_{1}^{2}$ in the defining representation of hs $[\lambda]$, we need the following matrix elements:

$$
\begin{aligned}
& V_{1,1}(p, q)=\left\langle 1\left|\left(V_{-1}^{2}\right)^{p}\left(V_{1}^{2}\right)^{q}\right| 1\right\rangle=\delta_{p, q} q ! \frac{\Gamma(q+\lambda+1)}{\Gamma(\lambda+1)} \\
& V_{2,2}(p, q)=\left\langle 2\left|\left(V_{-1}^{2}\right)^{p}\left(V_{1}^{2}\right)^{q}\right| 2\right\rangle=\delta_{p, q}(q+1) ! \frac{\Gamma(q+\lambda+2)}{\Gamma(\lambda+2)} \\
& V_{1,2}(p, q)=\left\langle 1\left|\left(V_{-1}^{2}\right)^{p}\left(V_{1}^{2}\right)^{q}\right| 2\right\rangle=-\delta_{p-1, q} \frac{(q+1) !}{\sqrt{-\lambda-1}} \frac{\Gamma(q+\lambda+2)}{\Gamma(\lambda+1)} \\
& V_{2,1}(p, q)=\left\langle 2\left|\left(V_{-1}^{2}\right)^{p}\left(V_{1}^{2}\right)^{q}\right| 1\right\rangle=\delta_{p, q-1} \frac{(p+1) !}{\sqrt{-\lambda-1}} \frac{\Gamma(p+\lambda+2)}{\Gamma(\lambda+1)}
\end{aligned}
$$

Plugging this expressions in the series expansion of C.2 we have for each matrix element:

$$
\langle i|\mathcal{O}| j\rangle=\sum_{m, n, p=0}^{\infty} \frac{\left(-e^{\rho} z\right)^{m}}{m !} \frac{\left(\mu e^{2 \rho} \bar{z}\right)^{n}}{n !} \frac{\left(e^{\rho} \bar{z}\right)^{p}}{p !} V_{i, j}(p, m+2 n),
$$


leading for $R_{\square}$ in the zero temperature (and large $\rho$ ) limit to:

$$
\begin{aligned}
\lim _{\substack{,, \bar{\tau} \rightarrow \infty \\
\text { fixed } \mu}} R_{\boxminus}= & \frac{1}{2}\left[\sum_{n=0}^{\infty}\left(\frac{\mu \bar{z}}{z^{2}}\right)^{n} \frac{\Gamma(2 n+1+\lambda)}{n ! \Gamma(1+\lambda)}\right]\left[\sum_{n=0}^{\infty}\left(\frac{\mu \bar{z}}{z^{2}}\right)^{n} \frac{(3+4 n+\lambda) \Gamma(2 n+2+\lambda)}{n ! \Gamma(2+\lambda)}\right]- \\
& \frac{1}{2}\left[\sum_{n=0}^{\infty}\left(\frac{\mu \bar{z}}{z^{2}}\right)^{n} \frac{\Gamma(2 n+2+\lambda)}{n ! \Gamma(2+\lambda)}\right]\left[\sum_{n=0}^{\infty}\left(\frac{\mu \bar{z}}{z^{2}}\right)^{n} \frac{(1+4 n+\lambda) \Gamma(2 n+1+\lambda)}{n ! \Gamma(1+\lambda)}\right] .
\end{aligned}
$$

After some simple manipulation, we recover the result (6.8).

\section{Eigenvalue of $V_{0}^{3}$ on the $K$-antisymmetric representation}

The result (7.5) can be derived as follows. We work at fixed $\lambda=-\mathcal{N}$ and from App. (A), we know that

$$
V_{0}^{3}=\frac{1}{12}\left[V_{-1}^{2},\left[V_{-1}^{2},\left(V_{1}^{2}\right)^{2}\right]\right]
$$

From this formula, we can compute the explicit matrix representation of $V_{0}^{3}$. It is a diagonal matrix with elements

$$
\left(V_{0}^{3}\right)_{n, n}=n^{2}-(\mathcal{N}+1) n+\frac{1}{6}(\mathcal{N}+1)(\mathcal{N}+2), \quad n=1, \ldots, \mathcal{N}
$$

The eigenvalue appearing on the r.h.s. of (7.5) is simply $\sum_{n=1}^{K}\left(V_{0}^{3}\right)_{n, n}$ and (7.5) is recovered upon use of $\sum_{n=1}^{K} n=\frac{1}{2} K(K+1)$ and $\sum_{n=1}^{K} n^{2}=\frac{1}{6} K(K+1)(2 K+1)$, and setting back $\mathcal{N}=-\lambda$.

\section{E The resummation functions $f_{N^{3} L O}$}

As discussed in Sec. (7.4), the function $f_{N^{3} L O, 1}^{(r)}$ depends non trivially on the scalar representation. We report here its expressions for the three considered representations.

$$
\begin{aligned}
r= & \square, \\
f_{N^{3} L O, 1}^{(r)}= & \frac{11 \mathcal{Z}^{2}}{16}+\frac{1}{2} \mathcal{Z}^{2} \cos \mathcal{Z}-\frac{13}{6} \mathcal{Z} \sin \mathcal{Z}-\frac{7 \cos \mathcal{Z}}{6} \\
& -\frac{29}{96} \cos (2 \mathcal{Z})-\frac{5}{24} \mathcal{Z} \sin \mathcal{Z} \cos \mathcal{Z}+\frac{47}{32}, \\
r= & \boxminus, \\
f_{N^{3} L O, 1}^{(r)}= & \frac{41 \mathcal{Z}^{2}}{8}+4 \mathcal{Z}^{2} \cos \mathcal{Z}-\frac{49}{3} \mathcal{Z} \sin \mathcal{Z}-\frac{25 \cos \mathcal{Z}}{3} \\
& -\frac{119}{48} \cos (2 \mathcal{Z})-\frac{23}{12} \mathcal{Z} \sin \mathcal{Z} \cos \mathcal{Z}+\frac{173}{16}, \\
r= & \boxminus, \\
f_{N^{3} L O, 1}^{(r)}= & \frac{273 \mathcal{Z}^{2}}{16}+\frac{27}{2} \mathcal{Z}^{2} \cos \mathcal{Z}-\frac{109}{2} \mathcal{Z} \sin \mathcal{Z}-\frac{53}{16} \mathcal{Z} \sin (2 \mathcal{Z}) \\
& -\frac{55 \cos \mathcal{Z}}{2}-\frac{269}{32} \cos (2 \mathcal{Z})+\frac{1149}{32} .
\end{aligned}
$$


The functions $f_{N^{3} L O, 2-3}$ are instead universal and read

$$
\begin{aligned}
f_{N^{3} L O, 2}= & -\frac{79 \mathcal{Z}^{3}}{192}-\frac{41}{96} \mathcal{Z}^{3} \cos \mathcal{Z}-\frac{7}{192} \mathcal{Z}^{3} \cos (2 \mathcal{Z})+\frac{57}{32} \mathcal{Z}^{2} \sin \mathcal{Z}+\frac{27}{64} \mathcal{Z}^{2} \sin (2 \mathcal{Z})-\frac{55 \mathcal{Z}}{32}+\frac{271 \sin \mathcal{Z}}{192} \\
& -\frac{29}{48} \sin (2 \mathcal{Z})-\frac{13}{192} \sin (3 \mathcal{Z})+\frac{19}{32} \mathcal{Z} \cos \mathcal{Z}+\frac{35}{32} \mathcal{Z} \cos (2 \mathcal{Z})+\frac{1}{32} \mathcal{Z} \cos (3 \mathcal{Z}), \\
f_{N^{3} L O, 3}= & \frac{17 \mathcal{Z}^{4}}{384}+\frac{167 \mathcal{Z}^{4} \cos \mathcal{Z}}{3072}+\frac{1}{96} \mathcal{Z}^{4} \cos (2 \mathcal{Z})+\frac{\mathcal{Z}^{4} \cos (3 \mathcal{Z})}{3072}-\frac{29}{128} \mathcal{Z}^{3} \sin \mathcal{Z}-\frac{19}{96} \mathcal{Z}^{3} \sin \mathcal{Z} \cos \mathcal{Z} \\
& -\frac{5}{384} \mathcal{Z}^{3} \sin \mathcal{Z} \cos (2 \mathcal{Z})+\frac{77 \mathcal{Z}^{2}}{288}+\frac{5}{384} \mathcal{Z}^{2} \cos \mathcal{Z}-\frac{23}{96} \mathcal{Z}^{2} \cos (2 \mathcal{Z})-\frac{47 \mathcal{Z}^{2} \cos (3 \mathcal{Z})}{1152} \\
& -\frac{109}{288} \mathcal{Z} \sin \mathcal{Z}-\frac{31 \cos \mathcal{Z}}{288}-\frac{41}{576} \cos (2 \mathcal{Z})+\frac{11}{288} \cos (3 \mathcal{Z})+\frac{7 \cos (4 \mathcal{Z})}{2304}+\frac{7}{32} \mathcal{Z} \sin \mathcal{Z} \cos \mathcal{Z} \\
& +\frac{5}{32} \mathcal{Z} \sin \mathcal{Z} \cos (2 \mathcal{Z})+\frac{1}{288} \mathcal{Z} \sin \mathcal{Z} \cos (3 \mathcal{Z})+\frac{317}{2304} .
\end{aligned}
$$

Open Access. This article is distributed under the terms of the Creative Commons Attribution License (CC-BY 4.0), which permits any use, distribution and reproduction in any medium, provided the original author(s) and source are credited.

\section{References}

[1] M.R. Gaberdiel and R. Gopakumar, An AdS 3 Dual for Minimal Model CFTs, Phys. Rev. D 83 (2011) 066007 [arXiv: 1011.2986] [INSPIRE].

[2] M.A. Vasiliev, Higher spin gauge theories in four-dimensions, three-dimensions and two-dimensions, Int. J. Mod. Phys. D 5 (1996) 763 [hep-th/9611024] [INSPIRE].

[3] M.A. Vasiliev, Higher spin matter interactions in (2+1)-dimensions, hep-th/9607135 [INSPIRE].

[4] M.R. Gaberdiel and R. Gopakumar, Triality in Minimal Model Holography, JHEP 07 (2012) 127 [arXiv: 1205.2472] [INSPIRE].

[5] M. Henneaux and S.-J. Rey, Nonlinear $W_{\infty}$ as Asymptotic Symmetry of Three-Dimensional Higher Spin Anti-de Sitter Gravity, JHEP 12 (2010) 007 [arXiv:1008.4579] [INSPIRE].

[6] A. Campoleoni, S. Fredenhagen, S. Pfenninger and S. Theisen, Asymptotic symmetries of three-dimensional gravity coupled to higher-spin fields, JHEP 11 (2010) 007 [arXiv: 1008.4744] [INSPIRE].

[7] M.R. Gaberdiel and T. Hartman, Symmetries of Holographic Minimal Models, JHEP 05 (2011) 031 [arXiv:1101.2910] [INSPIRE].

[8] M. Bañados, C. Teitelboim and J. Zanelli, The Black hole in three-dimensional space-time, Phys. Rev. Lett. 69 (1992) 1849 [hep-th/9204099] [INSPIRE].

[9] M. Bañados, M. Henneaux, C. Teitelboim and J. Zanelli, Geometry of the (2+1) black hole, Phys. Rev. D 48 (1993) 1506 [gr-qc/9302012] [inSPIRE].

[10] M. Ammon, M. Gutperle, P. Kraus and E. Perlmutter, Black holes in three dimensional higher spin gravity: A review, J. Phys. A 46 (2013) 214001 [arXiv:1208.5182] [INSPIRE].

[11] P. Kraus and E. Perlmutter, Partition functions of higher spin black holes and their CFT duals, JHEP 11 (2011) 061 [arXiv:1108.2567] [INSPIRE]. 
[12] M.R. Gaberdiel, T. Hartman and K. Jin, Higher Spin Black Holes from CFT, JHEP 04 (2012) 103 [arXiv: 1203.0015] [INSPIRE].

[13] P. Kraus and E. Perlmutter, Probing higher spin black holes, JHEP 02 (2013) 096 [arXiv: 1209.4937] [INSPIRE].

[14] M.R. Gaberdiel, K. Jin and E. Perlmutter, Probing higher spin black holes from CFT, JHEP 10 (2013) 045 [arXiv: 1307.2221] [INSPIRE].

[15] E. Hijano, P. Kraus and E. Perlmutter, Matching four-point functions in higher spin $A d S_{3} / C F T_{2}, J H E P 05$ (2013) 163 [arXiv:1302.6113] [INSPIRE].

[16] M. Beccaria and G. Macorini, On the partition functions of higher spin black holes, JHEP 12 (2013) 027 [arXiv: 1310.4410] [INSPIRE].

[17] E. Bergshoeff, M. Blencowe and K. Stelle, Area Preserving Diffeomorphisms and Higher Spin Algebra, Commun. Math. Phys. 128 (1990) 213.

[18] O. Coussaert, M. Henneaux and P. van Driel, The Asymptotic dynamics of three-dimensional Einstein gravity with a negative cosmological constant, Class. Quant. Grav. 12 (1995) 2961 [gr-qc/9506019] [INSPIRE].

[19] C. Pope, L. Romans and X. Shen, $W_{\infty}$ and the Racah-wigner Algebra, Nucl. Phys. B 339 (1990) 191 [INSPIRE].

[20] J. de Boer and J.I. Jottar, Thermodynamics of higher spin black holes in AdS $S_{3}$, JHEP 01 (2014) 023 [arXiv: 1302.0816] [INSPIRE].

[21] P. Kraus and T. Ugajin, An Entropy Formula for Higher Spin Black Holes via Conical Singularities, JHEP 05 (2013) 160 [arXiv:1302.1583] [INSPIRE].

[22] M.A. Vasiliev, Unfolded representation for relativistic equations in (2+1) anti-de Sitter space, Class. Quant. Grav. 11 (1994) 649 [INSPIRE].

[23] C.-M. Chang and X. Yin, Higher Spin Gravity with Matter in $A d S_{3}$ and Its CFT Dual, JHEP 10 (2012) 024 [arXiv:1106.2580] [InSPIRE].

[24] M. Ammon, P. Kraus and E. Perlmutter, Scalar fields and three-point functions in D=3 higher spin gravity, JHEP 07 (2012) 113 [arXiv:1111.3926] [INSPIRE]. 